\title{
Revestimentos Para Barreira Térmica: Evolução e Perspectivas
}

\author{
(Thermal Barrier Coatings: Evolution and Perspectives)
}

\author{
Carlos Roberto Camello Limarl \\ ${ }^{1}$ UNIMEP - Universidade Metodista de Piracicaba, Programa de Pós Graduação em Engenharia de Produção, Santa Bárbara \\ d'Oeste, São Paulo,Brasil.crclima@unimep.br
}

\begin{abstract}
Resumo
Há mais de quatro décadas, os revestimentos para barreira térmica, ou simplesmente TBC, vêm sendo decisivos para o desempenho de turbinas a gás no segmento aeronáutico e aeroespacial e, mais recentemente, também no desempenho de turbinas de geração de energia e motores diesel. Sua importância se sobrepõe aos desenvolvimentos conseguidos na área de materiais com o advento da solidificação unidirecional e a obtenção de monocristais. Durante todo este período, diversas pesquisas ao redor do mundo buscam compreender melhor os processos que ocorrem na interação dos próprios componentes metal-cerâmicos do sistema, tanto no seu processamento, como na operação dos componentes em serviço, sujeitos a distintas solicitações mecânicas e térmicas durante os ciclos de aquecimento e resfriamento dos componentes revestidos. Mais ainda, pesquisas buscam incessantemente melhorias de desempenho dos revestimentos que permitam atender as crescentes solicitações de aumento de temperatura de trabalho e resistência à agressão de agentes externos próprios do ambiente operacional de tais equipamentos. Este artigo mostra a importância dos revestimentos TBC, sua evolução recente, suas características principais, os diversos temas e objetos de investigação e os inúmeros desafios que ainda se apresentam, demandando mais pesquisa e abrindo opções de extrema relevância científica e tecnológica para investigação.
\end{abstract}

Palavras-chave: Revestimento para barreira térmica; TBC; Aspersão térmica; Metal-cerâmica.

\begin{abstract}
For over four decades, thermal barrier coatings, or simply TBC, have been decisive for the performance of gas turbines in aviation and aerospace, and more recently also in the performance of power generation turbines and diesel engines. Its importance overlaps the developments achieved in the area of materials with the advent of directional solidification and obtaining single crystals. Throughout this period, several studies around the world seek to better understand the processes occurring in the interaction of metalceramic system components themselves, both in their processing, as well as during operation of the components in service, subject to different mechanical and thermal stresses during cycles of heating and cooling of the coated components. Moreover, research constantly seek improvements in the performance of the coatings that allow meeting the growing demands of increased operating temperature and resistance to the aggression of external agents own the operating environment of such equipment. This article shows the importance of $T B C$ coatings, recent developments, its main features, the different subjects and objects of research and the many challenges that are still present, requiring more research and opening options of utmost importance to scientific and technological research.
\end{abstract}

Key-words: Thermal barrier coating; TBC; Thermal Spray; Metal-ceramic.

\section{Introdução}

Revestimentos para barreira térmica, mais conhecidos como TBC (Thermal Barrier Coatings), compõem uma das mais tradicionais e eficazes aplicações de revestimentos industriais. $\mathrm{O}$ conceito de TBC envolve a colocação de uma camada de isolante térmico entre o componente metálico e o gás quente da fonte de calor, para reduzir a transferência desse calor ao componente. Suas principais aplicações são em turbinas a gás e motores diesel, na indústria aeronáutica e automobilística,

Recebido em 18/12/2014, texto final em 18/12/2014. DOI: http://dx.doi.org/10.1590/0104-9224/SI1904.11 respectivamente, seguido por turbinas de geração de energia $[1,2,3]$. Atualmente, existe uma previsão de uso também em componentes fabricados de materiais compósitos de matriz cerâmica [4]. Um sistema de revestimento para barreira térmica consiste, tipicamente, de uma camada de ligação metálica (bond coat), em contato com o substrato metálico, e uma camada externa cerâmica (top coat), aplicada sobre a camada de ligação, conforme esquematizado na Figura 1 [2].

Em geral, os TBCs são aplicados em componentes que possuem canais internos de refrigeração a ar. Os projetos de TBCs consideram diversos fatores, como a configuração (geometria) e espessura do componente, fluxo de calor, coeficientes de transferência de calor e temperaturas na entrada da turbina e na combustão, e total de resfriamento de ar permitido. Adicionalmente, revestimentos mais finos são preferíveis para minimizar a adição de massa e o eventual fechamento dos canais de refrigeração [5]. 


\section{Camada Cerâmica (top coat)}

Camada de Ligação Metálica (bond coat)

\section{Substrato Metálico}

Figura 1. Esquema da estrutura típica de um sistema TBC [2].

\subsection{Componentes do sistema TBC}

A Figura 2 mostra a seção transversal de um TBC aplicado sobre um componente de turbina. De uma perspectiva de engenharia de materiais, deve-se considerar o TBC como um sistema integrado de materiais em vez de simplesmente um revestimento com material isolante térmico sobre um componente estrutural de superliga. A amostra representativa de um revestimento comercial, mostrado na Figura 3, ilustra a natureza de um revestimento de múltiplas camadas após exposição a alta temperatura.

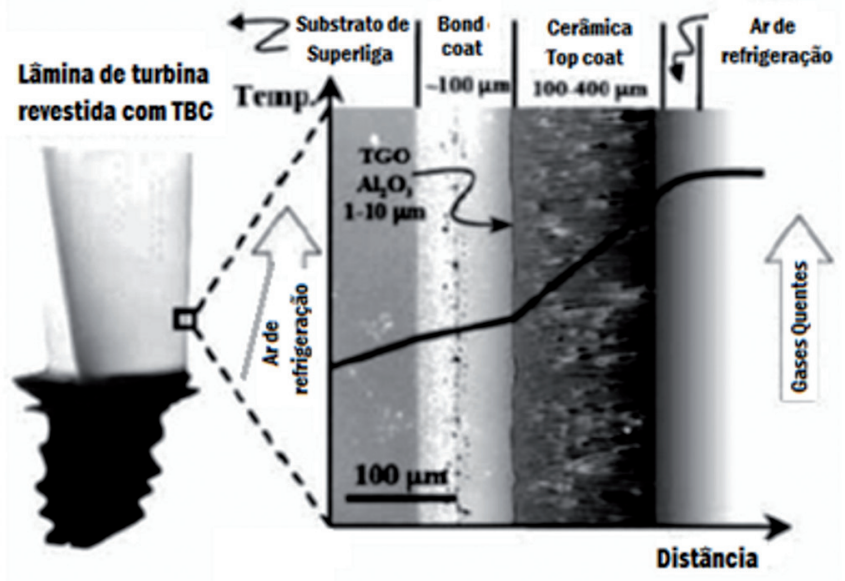

Figura 2. Esquema de um TBC para turbinas. A linha do gráfico representa o aumento da temperatura em função da distância em relação ao substrato [6].

Existem três principais camadas em adição à superliga e ao revestimento cerâmico de baixa condutividade. Entre a liga e o revestimento, está a camada de ligação (bond coat), assim chamado porque, nos estágios iniciais de desenvolvimento na produção de um revestimento viável, verificou-se que a superliga tinha de ser coberta com uma primeira camada metálica para garantir que o revestimento cerâmico se mantivesse aderente mediante oxidação. Entre a camada de ligação e o revestimento cerâmico, está a camada de óxido formada durante a exposição à alta temperatura, chamada de TGO (Thermally Grown Oxide) $[6,7,8]$. Finalmente, durante a formação dos revestimentos metálico e cerâmico, assim como posteriormente, durante o uso, forma-se uma camada de reação, como resultado da interdifusão entre a camada de ligação e a superliga [8].

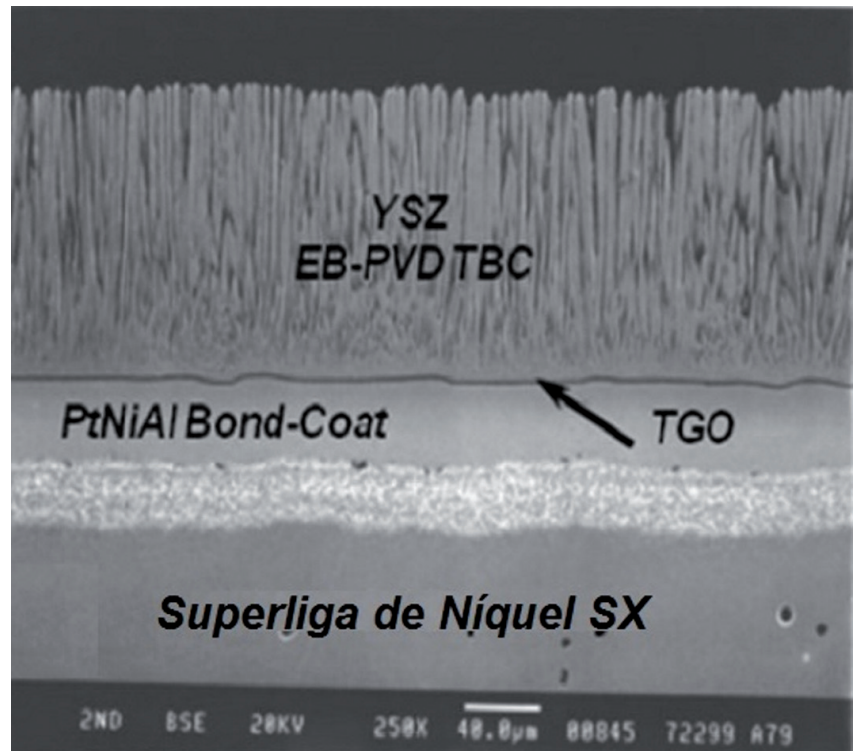

Figura 3. Vista de MEV da seção transversal de um TBC aplicado por EB-PVD, mostrando as diferentes camadas sobrepostas no sistema [6].

\subsection{Melhoramentos tecnológicos do sistema}

O desenvolvimento dos motores de turbinas a gás atuais resulta dos contínuos melhoramentos em diversos aspectos de engenharia, como projeto da turbina, aspectos de combustão e materiais envolvidos. Para que se tenha ideia destes melhoramentos substanciais nas últimas cinco décadas, devese citar o aumento na temperatura máxima do gás no aerofólio das turbinas permitido por tais melhorias. O aumento das temperaturas no aerofólio tem sido facilitado por três grandes desenvolvimentos: a) avanços dramáticos no projeto das ligas para produzir composições de ligas mais resistentes à fluência $\mathrm{e}$ à fadiga; b) avanços na tecnologia de fundição, que facilitou não somente a fundição de grandes lâminas e palhetas de superliga de monocristal (solidificação unidirecional), mas também os intrincados canais internos nas lâminas, projetados para facilitar o resfriamento e refrigeração; e c) o desenvolvimento de uma tecnologia de revestimento viável para depositar um revestimento isolante térmico nos componentes da turbina $[7,8]$.

Os dois primeiros itens são extensivamente tratados na literatura, mas o menos discutido ou conhecido é o desenvolvimento dos Revestimentos para Barreira Térmica, mesmo considerando que seu uso na última década permitiu um aumento muito grande nas temperaturas das turbinas, muito maior que a possibilitada pelas mudanças na tecnologia de solidificação direcional e técnicas de obtenção de ligas de monocristal dos últimos 30 anos $[7,8,9]$.

\section{Evolução dos processos de aplicação e estrutura dos re- vestimentos}

\subsection{Revestimentos cerâmicos}

Revestimentos protetores para componentes em superliga eram, inicialmente (seguramente há mais de cinco décadas), 
revestimentos de aluminetos simples, posteriormente modificados com silício, cromo e paládio. Depois de alguns anos, foram aplicados os primeiros revestimentos do tipo MCrAlY, onde M se refere a Ni, Co ou $\mathrm{Fe}$. Só bem posteriormente foram introduzidos os TBCs, revestimentos cerâmicos [9]. Existem, na atualidade, dezenas de patentes com variações do material MCrAlY original, largamente aplicados em revestimentos de engenharia na indústria aeroespacial e de turbinas de energia $[4,10]$.

Os primeiros estudos com revestimentos cerâmicos iniciaram-se na década de 1.970, com a aplicação por aspersão térmica a plasma de $\mathrm{MgO}-\mathrm{ZrO} 2$ sobre uma camada de ligação de NiCr. Este tipo de revestimento tinha como limitação a baixa vida útil devido à oxidação acelerada da camada de ligação. Melhores resultados foram obtidos para revestimentos cerâmicos de zircônia parcialmente estabilizada com $8 \%$ (em peso) de ítria, aplicado por aspersão térmica sobre uma camada de ligação de MCrAlY [9]. Mais recentemente, no início da década de 1980, os revestimentos cerâmicos à base de $\mathrm{ZrO}_{2}$ aplicados por deposição física de vapor (PVD) sobre uma camada de MCrAlY promoveram maior durabilidade, suficiente para serem usados em partes móveis de turbinas [6,9].

Os TBCs atuais consistem geralmente de um revestimento cerâmico de zircônia $\left(\mathrm{ZrO}_{2}\right)$ estabilizada com 6 a $8 \%$ de ítria $\left(\mathrm{Y}_{2} \mathrm{O}_{3}\right)$, em espessuras que variam, em geral, de 0,13 a $0,38 \mathrm{~mm}$. Aplicações mais recentes, como em turbinas para geração de energia, passam a requerer espessuras ainda maiores, de cerca de $1 \mathrm{~mm}[2,4,9]$. Esta camada cerâmica, muitas vezes referida simplesmente como YSZ (Ytria Stabilized Zirconia) ou mesmo PSZ (Partialy Stabilized Zirconia), é aplicada sobre uma camada de ligação metálica resistente à oxidação (bond coat), que, por sua vez, é aplicada sobre o componente de superliga à base de níquel. A espessura da camada de ligação varia normalmente entre 0,06 a $0,16 \mathrm{~mm}[2,4]$. Nas aplicações em motores diesel, onde as temperaturas são geralmente mais baixas, o revestimento cerâmico é muitas vezes aplicado diretamente sobre a superliga [9].

\subsection{Processos de aplicação e suas características}

Dois tipos principais de processo de aplicação dos revestimentos estão em uso, especialmente para a acamada cerâmica: para componentes relativamente pequenos, tais como as lâminas e pás de turbinas aeroespaciais, os revestimentos podem ser aplicados por deposição física de vapor por feixe de eletrons (EB-PVD - Electron Bean - Physical Vapor Deposition). Para os componentes maiores, como as câmaras de combustão e as lâminas e palhetas de geração de energia, e em turbinas estacionárias, os revestimentos são normalmente aplicados por aspersão térmica a plasma (APS - Air Plasma Spray) [11]. Em muitos aspectos, a escolha de materiais e sua produção representam uma tecnologia de materiais já bastante madura. Mas existe uma percepção de que novos sistemas TBC serão necessários para atender as demandas de turbinas de última geração atualmente sendo projetadas $[11,12]$.

A utilização de EB-PVD ou APS para aplicação dos revestimentos reflete diferentes abordagens para tolerância a deformação em serviço em função dos ciclos térmicos. A deposição durante o processo EB-PVD resulta na formação de uma estrutura colunar, como a mostrada na micrografia da Figura 3. Os contornos de grão promovem uma ligação fraca entre as colunas individuais monocristalinas, favorável à acomodação das tensões mecânicas geradas durante os ciclos térmicos [13]. A tolerância à deformação lateral resulta da estrutura colunar e lacunas entre colunas produzida pela rotação do componente durante a deposição. Imagens por microscopia electrônica de transmissão revelam que as colunas individuais também contém porosidade microscópica, que reduz a condutividade térmica do revestimento [5].

Diferentes métodos de aplicação das camadas de ligação foram desenvolvidos em grande parte para atingir as apertadas metas de produção na indústria. Normalmente, camadas de ligação de PtNiAl são formadas primeiro pela difusão ou eletrodeposição de Pt no componente de superliga e, em seguida, pela deposição física de vapor em atmosfera rica em alumínio. Nesta segunda etapa, o alumínio se difunde para a superfície da liga de níquel, enquanto este se difunde para fora, onde reage com o alumínio e platina para formar o revestimento de alumineto PtNiAl. Dependendo da qualidade do revestimento necessário, o alumínio é fornecido em um processo de empacotamento ou num reator de CVD de uma fonte de $\mathrm{AlCl}_{3}[5,6]$.

Em contraste, os revestimentos MCrAlY são geralmente depositados por um dos processos de aspersão térmica a plasma, em geral APS, ou plasma atmosférico. Estes processos são particularmente atraentes para o revestimento de grandes componentes e são, obviamente, mais baratos do que a deposição de EB-PVD. Além disso, como a aspersão por plasma não envolve um processo de difusão, revestimentos mais espessos podem ser depositados do que com o processo de aluminização utilizado para formar os revestimentos adesivos PtNiAl. Ainda, até os dias atuais, existe dúvida de qual destes tipos de revestimento é melhor para diferentes aplicações, principalmente em termos de desempenho, confiabilidade, durabilidade e custo. Em grande parte, isso é função de não se ter plenamente estabelecido a combinação das propriedades dos materiais que leva a mais longa vida em alta temperatura do revestimento.

\subsection{Evolução das ligas da camada de ligação}

Em revestimentos aspergidos por plasma (APS), a tolerância à deformação lateral e a reduzida condutividade térmica são conferidas pela incorporação de porosidade entre "panquecas" de material depositados sucessivamente. A Figura 4 mostra a seção transversal de um revestimento TBC aplicado por APS.

Duas classes principais de ligas para camada de ligação também evoluiram ao longo dos anos, ambas desenvolvidas com o objetivo de formar um óxido de alumínio $\left(\mathrm{Al}_{2} \mathrm{O}_{3}\right)$ por exposição ao ar a temperaturas elevadas. Isto é importante por diversas razões. Uma delas é que a $\mathrm{Al} 2 \mathrm{O} 3$ é compatível com a fase de YSZ, garantindo estabilidade termodinâmica de longo prazo ao revestimento. Sem revestimento, a maior parte das superligas à base de níquel forma complexos em várias camadas de óxido de níquel, espinelas de níquel-cromo e óxido de cromo, além de alumina, e estes não são termodinamicamente estáveis com YSZ $[2,4]$. 
Além disso, a alumina é geralmente considerada como sendo o óxido de alta temperatura de crescimento mais lento, em função da menor difusividade de oxigénio $[3,6]$. A seleção de ligas para camada de ligação é realmente um assunto complexo, mas o certo é que esta camada deve desempenhar um certo número de funções diferentes. Primordialmente, deve fornecer uma ligação entre a camada cerâmica e a liga do substrato. Nos primeiros dias de desenvolvimento de TBCs, tal ligação com a liga do substrato foi uma grande preocupação, especialmente quando aspergido por plasma, pois a zircônia depositada é um grande condutor de oxigênio e a camada de ligação deve também ser capaz de formar um óxido protetor, estável e de crescimento lento, para evitar ataque oxidativo da liga. Sabe-se que uma das principais formas de falha é associada com a falha do óxido de alumínio de protecção formado sobre a liga metálica da camada de ligação, o chamado TGO [5, 14]. Assim, a camada de ligação deve também ter estabilidade morfológica suficiente para que, durante aquecimento e resfriamento, bem como a alta temperatura, não cause distorções ou introduza defeitos de interface.

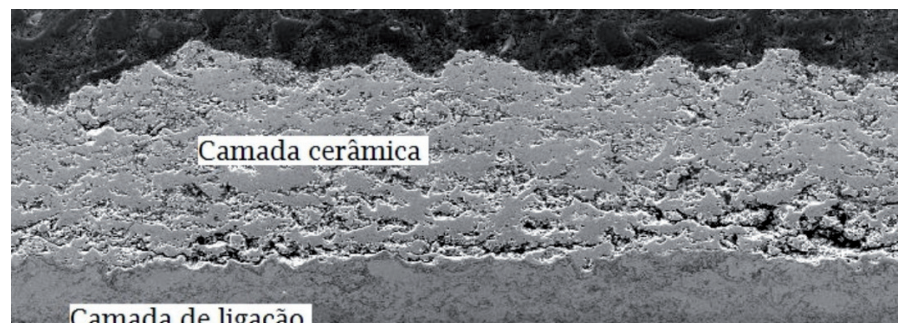

Camada de ligação

Substrato

$2 \mathrm{G} \times \mathrm{U}$

$x 15 \mathrm{G}$

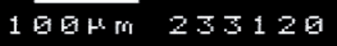

Figura 4. Imagem de MEV da seção transversal de um revestimento para barreira térmica (TBC) aplicado por APS Air Plasma Spray sobre substrato metálico.

As duas classes de ligas para camada de ligação que foram desenvolvidas com sucesso na aplicação prática são o aluminido de níquel modificado com platina (PtNiAl) e ligas MCrAlY (M se referindo a um ou mais dos elementos $\mathrm{Co}, \mathrm{Ni}$ e Fe) [6]. A escolha destas duas classes de ligas é, em grande parte, baseada no seu uso anterior como ligas resistentes à oxidação e corrosão, revestimentos usados para proteger ligas de alta temperatura antes do advento dos TBCs. Por exemplo, PtNiAl foi originalmente desenvolvido como um revestimento resistente à oxidação como alternativa para proteger ligas em funcionamento a uma temperatura superior àquelas possíveis com as ligas MCrAlY disponíveis naquele momento [5].

Para proporcionar um maior reservatório de alumínio para alimentar a TGO, seria de se esperar uma camada de ligação mais espessa e, quanto maior o seu teor de alumínio, melhor. No entanto, espera-se também que esta camada tenha o mais elevado limite de elasticidade possível a alta temperatura, e a maior compatibilidade possível de expansão térmica com a superliga, para que não surjam problemas maiores de tensões durante os ciclos térmicos experimentados em serviço [15]. Uma imagem típica de uma camada TGO é apresentada na Figura 5. De certa forma, estas propriedades se tornam muitas vezes difíceis de conciliar, favorecendo um ou outro tipo de liga.

Inúmeras composições de ligas McrAlY têm sido propostas e estão disponíveis comercialmente na busca de melhorias no desempenho das camadas de ligação [16, 17], assim como variações nos processos de aplicação destas camadas com o mesmo propósito $[6,18]$, temas que estarão presentes nas discussões das próximas seções.

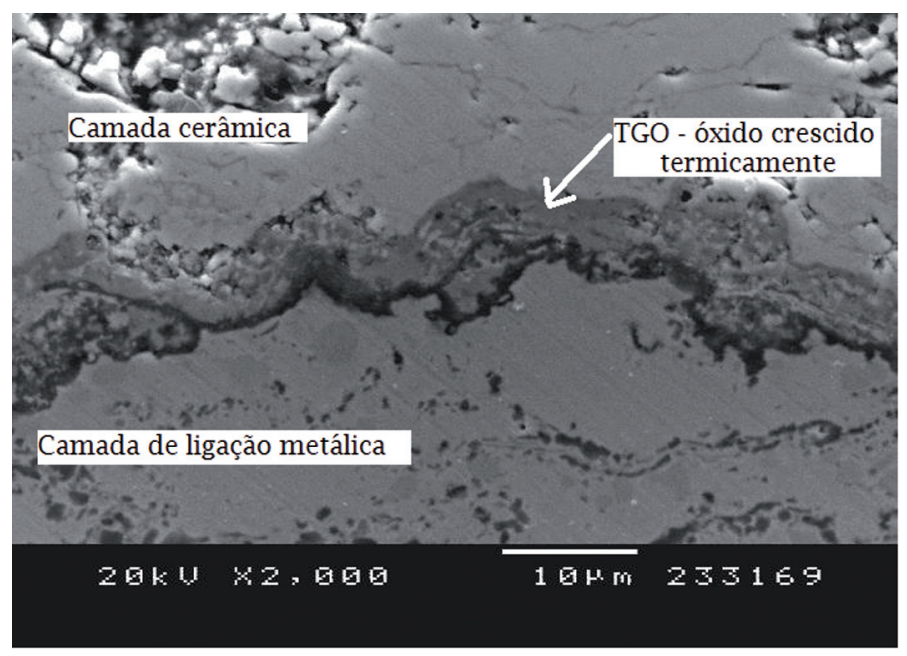

Figura 5. Imagem de MEV da secção transversal de um TBC aplicado por APS após ciclo térmico mostrando a camada de óxido formada ou TGO.

\section{Desenvolvimentos, desafios e perspectivas}

A capacidade de isolamento térmico de um TBC é incontestável. Entretanto, sua durabilidade e suas propriedades mecânicas variam fortemente com diversos fatores, incluindo condições de processo, condições operacionais em serviço, materiais envolvidos e pré ou pós-tratamentos. Geralmente, a falha de um TBC é por delaminação nas seções da camada cerâmica, separadas da camada de ligação. Isto ocorre devido à formação de trincas na camada cerâmica e na interface metal-cerâmica com a camada de ligação (defeitos microestruturais). As trincas são devidas às tensões residuais provocadas pela oxidação da camada de ligação metálica em altas temperaturas (formação e crescimento da TGO), devido à permeabilidade da camada cerâmica porosa, e às diferenças de expansão térmica cíclica (fadiga térmica no aquecimento e resfriamento dos componentes, durante o serviço), resultantes da diferença de cerca de $30 \%$ no coeficiente de expansão térmica entre a zircônia parcialmente estabilizada e a camada de ligação metálica $[2,3,4]$.

Diversas pesquisas têm sido realizadas na busca de solucionar estes problemas, envolvendo, inclusive, o conceito de revestimentos graduados $[19,20]$, pré ou pós-tratamentos dos revestimentos [21, 22, 23] e adição de dopantes [24, 25]. 
Entretanto, os resultados não são considerados conclusivos ou definitivos, principalmente em virtude da peculiaridade dos métodosdetrabalhoeavaliaçãodecadapesquisa, individualmente. Além disso, o crescente desafio de novas condições de serviço em mais altas temperaturas e distintas atmosferas faz com que as pesquisas tenham que seguir evoluindo, e qualquer melhoria relacionada ao desempenho em serviço ou aumento do ciclo de vida de TBC's pressupõe valiosa contribuição tecnológica e científica. Assim, processos, materiais e sistemas consolidados são levados a novos patamares de solicitação, exigindo novos desenvolvimentos, em diversas áreas do conhecimento e em diversas características dos revestimentos para barreira térmica.

\subsection{Influência das Tensões Residuais}

$\mathrm{O}$ estado de tensões nos revestimentos para barreira térmica advém, especialmente, da diferença entre os coeficientes de expansão térmica dos materias metálicos e cerâmicos envolvidos. A tensão residual definitiva dos revestimentos é uma combinação da tensão desenvolvida durante a deposição (dependendo do estado da partícula, temperatura do substrato, etc.) e a dependente térmica, referente às diferenças de coeficientes de expansão térmica, aos gradientes de temperatura e rigidez do revestimento/ substrato. Durante os ciclos térmicos de serviço, também existem tensões resultantes da oxidação da camada de ligação, que será discutida em outra seção. Há, ainda a componente relacionada a mudanças na microestrutura da interface, devido a transformações de fase, difusão no estado sólido, crescimento de grão, precipitação, segregação, coalescência de partículas de segunda fase e rearranjo de discordâncias [26].

Sob ciclos de temperatura em serviço, as tensões residuais ativam mecanismos inelásticos que, por sua vez, limitam a durabilidade do revestimento, levando-o à fratura [27]. É importante lembrar que as tensões e deformações influenciam diferentemente cada camada do revestimento. É menos importante no caso da camada externa cerâmica, pois esta não precisa ser densa, visto que sua função é isolar a liga subjacente (porosidade favorece o isolamento) e não fornece proteção contra oxidação. É projetada com uma microestrutura espacialmente configurada quanto à porosidade, a qual proporciona baixa rigidez no plano e tolerância à deformação [28-32]. Já a camada de ligação precisa ser densa para exercer sua função (mínima porosidade). A Figura 6 ilustra a porosidade típica de uma camada cerâmica de YSZ aplicada por APS.

Alguns estudos sobre mecanismos de degradação a alta temperatura de TBC constituído de uma base Ni22Cr10Al1Y, com $0,15 \mathrm{~mm}$ de espessura e uma camada externa de $0,22 \mathrm{~mm}$ de PSZ sobre substrato superliga MAR-M-247 concluíram que as tensões provenientes da diferença de coeficientes de expansão térmica entre os materiais envolvidos, só resultaram em falha do revestimento quando combinados com oxidação da camada de ligação [33].

Revestimentos aspergidos aplicados por Cold Gas Spray (CGS), assim como por HVOF, apresentam, em geral, tensões residuais compressivas, devido à pressão de impacto das partículas ser bastante elevada em função da elevada velocidade

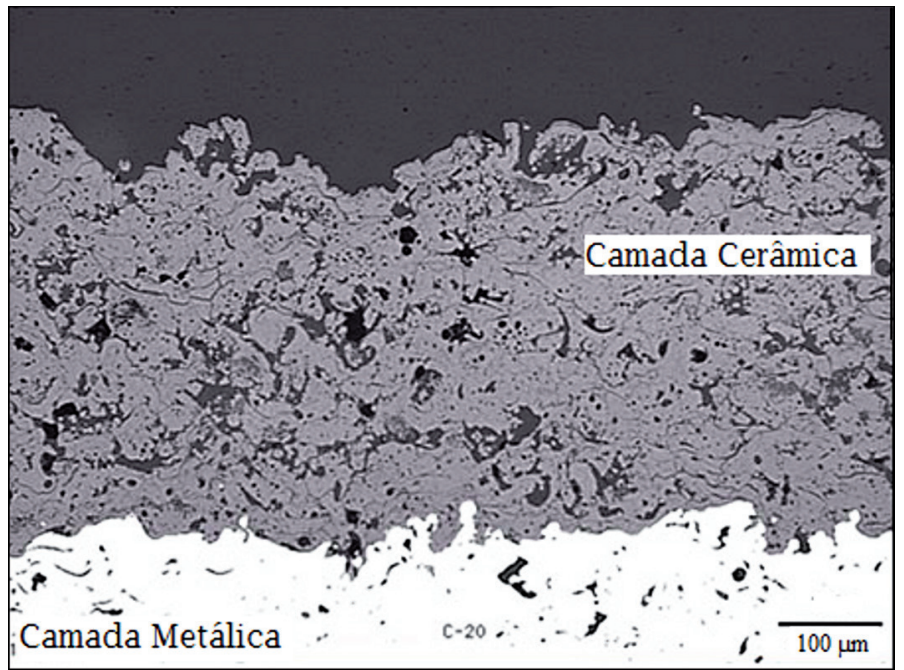

Figura 6. Imagem de MEV da seção transversal de um TBC mostrando a porosidade de cerca de 15 \% na camada cerâmica.

de saída das partículas da tocha, em torno de $900 \mathrm{~m} / \mathrm{s}$ para o HVOF e de até $1.200 \mathrm{~m} / \mathrm{s}$ para o CGS [2,34]. Em função de tais características, diversas pesquisas têm sido realizadas utilizando estes métodos de aplicação [35-38]. Os resultados são, em geral, promissores, encontrando aplicação rela em diversos segmentos.

Estudos das tensões residuais em sistemas TBC com camada externa de YSZ, realizados por simulação usando o Método de Elementos Finitos (FEM), concluíram que existe um elevado gradiente térmico na camada cerâmica durante o período inicial de resfriamento, o qual aumenta com o aumento da taxa de resfriamento. Adicionalmente, na ocorrência de ciclos térmicos (típicos do processo produtivo), a taxa de resfriamento é crucial na determinação da vida dos TBC's [39].

Pesquisas estudando aplicações de TBCs em motores diesel, apresentaram, há mais de vinte anos, tentativas de aprimorar o controle de tensões residuais através da temperatura de aplicação e do uso de revestimentos estruturados com camada de base metálica (NiCrAlY), duas camadas de compósitos (metal-zircônia) e uma camada externa cerâmica (zircônia). As espessuras totais destes revestimentos, em pesquisa, chegavam a 2,5 $\mathrm{mm}$ [40]. As tensões residuais foram reduzidas, retardando a delaminação eventual do revestimento.

Outras pesquisas [41] descreveram, também há cerca de duas décadas, revestimentos graduados para barreira térmica utilizados em selos de ar de turbinas, composto de uma camada de ligação metálica, uma camada de compósito (metal + alumina), uma camada graduada de metal para alumina, outra camada de compósito (alumina + metal), uma segunda camada com mistura cerâmica (alumina +zircônia), uma camada de cerâmica (zircônia completamente estabilizada), uma terceira camada mista cerâmica (zircônia + alumina) e, finalmente, uma camada cerâmica de zircônia parcialmente estabilizada. Segundo os autores, este revestimento com estrutura mista pode reduzir tensões devidas a diferenças de expansão térmica entre metais e cerâmicas, e minimizar o trincamento, melhorando a resistência à delaminação.

Outros pesquisadores conseguiram, ainda, uma redução das 
tensões residuais na interface entre a camada metálica de base e a camada cerâmica, através do resfriamento do substrato para uma faixa entre $35^{\circ} \mathrm{C}$ e $70^{\circ} \mathrm{C}$, durante a aspersão térmica, num procedimento denominado ATCS (aspersão com atmosfera e pressão controladas) [42].

Lima e Trevisan [20] estudaram um revestimento para barreira térmica com graduação dos pós metálico e cerâmico, sem utilização de bond-coat metálico convencional, eliminando a interface abrupta metal-cerâmica entre a camada de ligação metálica e a camada externa cerâmica, típica dos sistemas duplex. Como resultado, o sistema com graduação de mistura $25 \%$ metal - 75\% cerâmica na interface com o substrato metálico apresentou resultados bem próximos ao sistema convencional duplex, quando avaliado pela resistência adesiva, dureza, microestrutura e estabilidade de fases, em espessuras de até $300 \mu \mathrm{m}$. Como ponto de melhoria, a permeabilidade do revestimento, em função da porosidade da cerâmica em toda a espessura, poderia levar a problemas de oxidação nas interfaces metal-cerâmicas, comprometendo a durabilidade do revestimento em condições operacionais.

Xie et al. [43] desenvolveram estudo sobre o efeito do jateamento da camada de ligação na durabilidade de revestimentos para barreira térmica com base nas tensões residuais desenvolvidas durante este processo. Segundo os autores, os resultados são positivos, melhorando o comportamento dos revestimentos sob tensões térmicas e sua vida útil.

Bach et. al. [44] estudaram a aplicação de revestimentos de MCrAlY pelo processo HVOF em substituição ao processo LPPS (Low Pressure Plasma Spray) para aplicações de proteção contra corrosão a quente em lâminas de turbinas. Utilizaram diversos equipamentos e concluíram que há viabilidade e grande potencial para uso deste processo na aplicação deste tipo de revestimento, mostrando as limitações de seu estudo em relação às condições de avaliação dos revestimentos em serviço, mas percebendo a importância da alta velocidade das partículas e da quantidade de calor nas mesmas quando da adesão ao substrato, favorecendo o desempenho do revestimento obtido.

Pesquisa realizada em TBCs com camada de ligação aplicada por HVOF avaliou as tensões residuais utilizando o Método Modificado de Remoção de Camadas (MLRM), então em estudo avançado nos grupos da ASM International. No estudo foi verificado que as tensões residuais foram influenciadas principalmente pela história térmica das partículas individuais e pela deformação plástica dos depósitos cerâmicos [45].

É importante observar que a avaliação das tensões residuais nos revestimentos é realizada por distintas técnicas e procedimentos em diversas pesquisas, utilizando-se desde indentação, difração de raios $\mathrm{X}$, até métodos de remoção de camadas, variando assim a dimensão do objeto de estudo.

\subsection{O papel da porosidade}

A porosidade é inerente aos revestimentos executados por aspersão térmica. Os valores de porosidade podem variar bastante, desde valores menores do que $1 \%$, até valores acima de $15 \%$, dependendo do processo de deposição, do material vaporizado e condições operacionais. A porosidade pode ser interligada e se estender até a superfície do substrato. Os selantes são utilizados como pós-tratamento para preencher esses poros, quando indesejados. No caso dos TBC's, a porosidade da camada cerâmica é desejável, pois auxilia em sua função de isolamento térmico. Mas tem sua influência também em relação ao desempenho estrutural do revestimento, influenciando em sua tolerância à deformação.

Neuer et al. [46], assim como Moskal et al. [47] salientam a importância de se considerar, no projeto de revestimentos para barreira térmica, a influência do nível de porosidade sobre a condutividade térmica do revestimento, em contraposição ao seu efeito sobre as propriedades de adesão e resistência à corrosão, pois aumentando o nível de porosidade, diminui a condutividade térmica, que é a função principal deste tipo de revestimento. Segundo os autores, em altas temperaturas, efeitos de sinterização das microtrincas e vazios entre partículas devem ser considerados, pois aumentam a condutividade térmica, prejudicando a função principal de isolamento do TBC.

Steffens et al. [48] discutiram, há mais de vinte anos, a instabilidade da condutividade térmica sob condições de trabalho, devido à porosidade. Concluíram que a graduação da porosidade da camada de zircônia parcialmente estabilizada (mais porosa na ligação com a camada de base e menos porosa na superfície externa do revestimento), conseguida através da variação da velocidade e/ou distância de aspersão, contribui bastante para a resistência ao choque térmico.

Em um estudo com o uso de pós-tratamento com laser de $\mathrm{CO}_{2}$ pulsado sobre revestimentos cerâmicos de $\mathrm{ZrO}_{2} 12 \mathrm{wt} \% \mathrm{Y}_{2} \mathrm{O}_{3}$, Tsai e Tsai [49] concluíram que a grande contribuição deste processo de pós-tratamento para o aumento de vida de TBC's está na melhoria da acomodação de tensões, ocorridas durante o ciclo térmico, típico de componentes onde se aplica TBC', através das trincas segmentadas que são produzidas durante a formação de uma microestrutura colunar, sendo estas trincas perpendiculares à superfície da camada cerâmica. A expectativa nestes casos é que, selando os poros e microtrincas apenas próximo à superfície do revestimento, seria possível reduzir a permeabilidade a gases mantendo boas características térmicas e mecânicas para o revestimento. Porém, estas tentativas produzem normalmente uma morfologia superficial tipo "lama seca", com uma rede de trincas devido à contração de solidificação da zircônia densificada, prejudicial ao revestimento, tanto mecânica, quanto em relação à permeabilidade.

Em pesquisas realizadas utilizando camada de ligação de NiCoCrAlY e YSZ aplicados por APS, Swadzba et al. [50] aplicaram os revestimentos variando a porosidade e a composição química do sistema em quatro gradientes distintos. Concluíram que a graduação da composição química não levou a qualquer melhoria na vida do revestimento, diferentemente do ocorrido com a graduação da porosidade, que levou a aumento da vida do revestimento por ser mais efetiva na acomodação de tensões residuais.

\subsection{Resistência adesiva do revestimento}

A adesão ou aderência entre as interfaces camada de ligação/ substrato e camada cerâmica/camada de ligação afeta 
diretamente a qualidade e, ainda mais, o desempenho dos TBC's. $\mathrm{O}$ intertravamento mecânico está diretamente relacionado a algumas pré-condições para a obtenção de um processo de aspersão térmica efetivo, como a rugosidade da superfície, a limpeza da superfície do substrato, a força de adesão dos revestimentos, a morfologia de adesão das gotas individuais (splats) após o processo de revestimento, tipo e parâmetros do processo de aspersão. Assim, o intertravamento mecânico varia dependendo dos efeitos físicos e químicos [2, 51].

Pesquisas realizadas sobre a característica de deformação de TBCs aplicados por plasma, utilizando indentação hertziana e flexão a quatro pontos, com monitoramento por emissão acústica verificou que as tensões e cargas que levam à falha do revestimento foram significantemente influenciadas pelo resfriamento externo (por ar comprimido) aplicado ao substrato e pelo procedimento de aplicação, em especial o tempo entre a aplicação de camadas sucessivas do revestimento. Também ficou evidenciada a importância do desempenho da camada de ligação para a melhoria da adesão mecânicado revestimento [52].

No desenvolvimento de trabalhos de pesquisa utilizando HVOF para aplicação da camada de ligação de um TBC de YSZ, foram avaliados o efeito da rugosidade superficial, do tamanho das partículas e da expansão térmica sobre a adesão dos revestimentos, avaliados pelo ensaio de tração da norma ASTM C633 (Tensile Adhesion Test). Os menores valores de adesão foram relacionados à fratura na interface camada de ligação/ camada cerâmica, provavelmente relacionados às tensões residuais na interface metal-cerâmica. Para valores de adesão das amostras individuais, os maiores valores de adesão foram relacionados às amostras com menor rugosidade superficial [53].

Tal resultado se contrapõe ao de Yamazaki e colaboradores [54] que estudaram o efeito da rugosidade interfacial da camada de ligação sobre a adesão de TBC's aplicados por plasma, tanto na camada de base, quanto na cerâmica, após ciclo térmico. Os autores concluíram que quanto mais lisa a interface, menor a adesão residual após o ciclo térmico de operação, levando ao destacamento do revestimento.

Knight et al. [55], estudando TBC's com camada de ligação à base de $\mathrm{Co}$, concluíram que a rugosidade superficial da camada de ligação influencia no desempenho do revestimento ao choque térmico e ao ciclo térmico, sendo definida pelo processo e condições de aplicação, podendo ser também correlacionada à resistência adesiva do revestimento. Porém, nestes dois últimos trabalhos, as condições de processo e materiais foram distintos dos aplicados no trabalho anterior [53], o que pode explicar as diferenças de resultados.

\subsection{Oxidação em alta temperatura e outros fatores adicionais}

Outras pesquisas testaram cold spray na aplicação de liga CoNiCrAlY, utilizada como camada de ligação em TBCs, estudando o comportamento da liga quanto à oxidação em alta temperatura, comparativamente ao mesmo material aplicado por aspersão a plasma convencional (APS) e plasma de baixa pressão (LPPS). Os autores concluíram que o revestimento aplicado por cold spray apresentou muito menor oxidação e nível de tensões residuais que os revestimentos aplicados por plasma [56].
Limarga et al. [19] estudaram, no conceito de FGM ou Functionally Graded Materials, alumina como inter-camada para melhorar a resistência à oxidação de um sistema TBC devido a sua baixa difusividade de oxigênio. Os resultados mostraram melhorias nas propriedades mecânicas e resistência à oxidação, porém com pequena redução no efeito de isolamento térmico.

Outra linha de pesquisa refere-se ao estudo da aplicação de camada intermediária de $\mathrm{Cr}_{3} \mathrm{C}_{2}$ por PVD entre a camada de ligação e a camada cerâmica (interlayer) com o intuito de modificar a dinâmica de formação e crescimento da chamada TGO (Thermally Grown Oxide). Em tais trabalhos, foram desenvolvidos experimentos em aplicações de TBC's com camada de base aplicada por HVOF e utilizando diferentes materiais como bond coat. Como resultados principais, os dois materiais usados não apresentaram diferenças significativas de desempenho nos testes de oxidação isotérmica e, mais importante, a aplicação da camada intermediária sugere melhorias na resistência à oxidação da camada de ligação e modificação nas tensões residuais por preencher imperfeições superficiais e alterar a cinética de oxidação desta camada de ligação [57].

Como a oxidação da camada de ligação, típica dos TBC's em serviço, afeta especialmente a vida dos revestimentos, causando sua delaminação, diversos estudos vêm sendo atualmente efetuados nesta linha, buscando maior entendimento do processo em si e da variação ocasionada por este processo nas tensões e morfologia da interface [35, 58-64].

\subsection{Outros fatores de melhoria de desempenho}

Diversas pesquisas vêm sendo realizadas também na busca de alternativas à ZircÓnia parcialmente estabilizada (YSZ), especialmente objetivando redução na condutividade térmica do TBC, ou seja, maior capacidade de isolamento térmico do revestimento para mais altas temperaturas de trabalho [65-68]. O desafio maior é encontrar alternativas que ofereçam a mesma condição de comportamento mecânico do revestimento YSZ.

Matsumoto [69], estudando desenvolvimentos em TBC's, sugere que pequenas adições de $\mathrm{La}_{2} \mathrm{O}_{3}$ mostram-se efetivas na redução da condutividade térmica da camada cerâmica de Zircônia aspergida por plasma. Porém, não há referência ao comportamento e propriedades mecânicas do revestimento.

Racek e Berndt [70] estudaram as variações das propriedades mecânicas dentro de revestimentos para barreira térmica de Zircônia naonoestruturada, principalmente usando técnicas de indentação pela espessura da camada do revestimento. Concluíram que existe variação do módulo de elasticidade em relação aos revestimentos microestruturados, influenciando o desempenho e durabilidade. Não discutem, porém, a condutividade térmica.

Recentemente, Maurel e coautores [71], estudando a influência do nível de deformações localizadas nos cristais do substrato e a falha por delaminação de TBC's, concluíram que a anisotropia do substrato influencia o nível de deformações localizadas e, consequentemente, a vida do revestimento.

Um grande esforço de pesquisa vem sendo direcionado 
recentemente ao estudo do ataque por depósitos fundidos sobre a camada cerâmica dos TBC's, especialmente o ataque por silicatos fundidos, conhecidos como CMAS (CalciumMagnesium-Alumino-Silicate) $[9,72,73]$. Este ataque ocorre em função da ingestão de finas partículas provenientes do ambiente, como areia, cinza vulcânica, etc. $[74,75]$ e afeta diretamente o desempenho dos revestimentos e, consequentemente, das turbinas em altas temperaturas.

Diversas pesquisas continuam a ser desenvolvidas em todo o mundo na busca de atender as demandas da área aeroespacial e automotiva, principais usuários dos revestimentos para barreira térmica [76, 77]. Cada pequeno avanço é significativo, não existindo situação definitiva. Na medida em que novos materiais são propostos para as camadas cerâmicas e metálicas de TBC's [17, 78], em conjunção com a evolução dos processos de aplicação [79] e sistemas de controle e monitoramento [80], abrem-se as portas para novos projetos de pesquisa, em diversos aspectos deste mesmo tema.

\section{Considerações finais}

Os revestimentos para barreira térmica continuam sendo objeto de diversas pesquisas em todo o mundo, que buscam e oferecem novas soluções para cada nova situação proposta, além de buscar melhorias de desempenho, confiabilidade e durabilidade dos revestimentos convencionais.

Os temas aqui discutidos refletem os desafios colocados na busca de sistemas alternativos ou mesmo de melhoria dos sistemas existentes, em função da sinergia entre os diversos elementos que compõem os sistemas TBC e da ampla gama de possibilidades oferecidas pela oferta de produtos e processos para sua aplicação.

\section{Referências Bibliográficas}

[1] HANGONG, W.; LIUYING, W. Thermal spraying application and development trends. Thermal Spray connects: explore its surfacing potential. Proceedings of the ITSC 2005, E. Lugscheider Ed., ASM International, 2005.

[2] LIMA, C.R.C., TREVISAN, R.E. Aspersão Térmica: Fundamentos e Aplicações. $2^{\text {a }}$ Ed., Art Liber, São Paulo, 2007

[3] PAWLOWSKI, L. The Science and Engineering of Thermal Spray Coatings. John Wiley \& Sons, New York, NY, 1995.

[4] DAVIS, J.R. (Ed.). Handbook of Thermal Spray Technology. New York, ASM International, 2004, 338 p.

[5] D.R. CLARKE AND C.G. LEVI Materials Design for the Next Generation Thermal Barrier Coatings Annual Review Materials Research, v. 33, 2003, p.383-417.

[6] DAROLIA, R. Thermal barrier coatings technology: critical review, progress update, remaining challenges and prospects. International Materials Review, v. 58, n.6, 2013, p. 315-347.

[7] RAJENDRAN, R. Gas turbine coatings - An overview. Engineering Failure Analysis, v. 26, 2012, p. 355-369.

[8] W.R. CHEN, X. WU, B.R. MARPLE, P.C. PATNAIK The growth and influence of thermally grown oxide in a thermal barrier coating. Surface and Coatings Technology, 201 (2006) 1074-1079

[9] CLARKE, D.R., OECHSNER, M., PADTURE, N.P. Thermal-barrier coatings for more efficient gas-turbine engines. MRS Bulletin, v. 37, n.10, p.891-899, 2012.

[10] ALMEIDA, D.S. Estudo de revestimentos cerâmicos sobre substrato metálico, obtidos por deposição física de vapores por feixe de elétrons para aplicação como barreira térmica. Tese (Doutorado), Engenharia e Tecnologia Espaciais/Ciência e Tecnologia de Materiais e Sensores, INPE - Instituto Nacional de Pesquisa Espaciais, S. José dos Campos, SP, 2005.

[11] OLIVEIRA, J.P., DUARTE, J.F. Revestimentos cerâmicos utilizados como barreira térmica. Cerâmica, v. 59, p. 186-191, 2013.

[12] GOSWAMI, B. RAY, A.K. SAHAY, S.K. Thermal Barrier Coating System for Gas Turbine Application: A Review. High Temperature Materials and Processes, v. 23, n.2, p. 73-92, 2004. [13] BEELE, W., MARIJNISSEN, G., LIESHOUT A.V. The evolution of thermal barrier coatings-status and upcoming solutions for today's key issues. Surface and Coatings Technology, v. 120-121, p. 61-67, 1999.

[14] CHEN, W.R., WU, X., MARPLE, B.R., PATNAIK, P.C. The growth and influence of thermally grown oxide in a thermal barrier coating. Surface \& Coatings Technology, v. 201, p. 1074-1079, 2006.

[15] KARLSSON, A. M., HUTCHINSON, J.W., EVANS, A.G. The displacement of the thermally grown oxide in thermal barrier systems upon temperature cycling. Materials Science and Engineering, A, v. 351, p.244-257, 2003.

[16] HAYNES, J.A., FERBER, M.K., PORTER W.D. Thermal Cycling Behavior of Plasma-Sprayed Thermal Barrier Coatings with Various MCrAIX Bond Coats. Journal of Thermal Spray Technology, v. 9, n. 1, 38-48, 2000.

[17] LEVI, C. G. Emerging materials and processes for thermal barrier systems. Current Opinion in Solid State and Materials Science, v. 8, p. 77-91, 2004.

[18] RICHER, P., YANDOUZI, M., BEAUVAIS, L., JODOIN, B. Oxidation behaviour of CoNiCrAlY bond coats produced by plasma, HVOF and cold gas dynamic spraying. Surface \& Coatings Technology, v. 204, p. 3962-3974, 2010.

[19] LIMARGA, A. M.; WIDJAJAB, S.; YIP, T.H. Mechanical properties and oxidation resistance of plasma-sprayed multilayered A12O3/ZrO2 thermal barrier coatings. Surface and Coatings Technology, v.197, p. 93-102, 2005.

[20] LIMA, C.R.C., TREVISAN, R. E. Graded plasma spraying of premixed metal-ceramic powders on metallic substrates. Journal of Thermal Spray Technology, v.6, n.2, p. 199-204, 1997.

[21] KOBYLAÑSKA-SZKARADEK, K. Thermal barrier $\mathrm{ZrO} 2$ - Y2O3 obtained by plasma spraying method and laser melting. Archives of Materials Science and Engineering, v. 36, n.1, p. 12-19, 2009.

[22] ABDULLAH C. KARAOGLANLI, A.C., DIKICI,H., KUCUK, Y. Effects of heat treatment on adhesion strength of thermal barrier coating systems. Engineering Failure Analysis, v. 32, p. 16-22, 2013.

[23] RICO, A., SEVILLANO, F., MÚNEZ, C.J., LÓPEZ, M.D., 
UTRILlA, V., RODRÍGUEZ, J., POZA, P. Effect of laser remelting on the tribological performance of thermal barrier coatings. Journal of Nanoscience and Nanotechnology, v. 12, n. 6, p. 4984-4990, 2012.

[24] ALMEIDA, D.S., CAIRO, C.A.A., SILVA, C.R.M., NONO, M.C.A. Thermal barrier coating by electron beamphysical vapor deposition of zirconia co-doped with yttria and niobia. Journal of Aerospace Technology and Management, v. 2, n.2, p. 195-202, 2010.

[25] BANSAL, N.P., ZHU, D. Effects of doping on thermal conductivity of pyrochlore oxides for advanced thermal barrier coatings. Materials Science and Engineering: A, v. 459, n. 1-2, p.192-195, 2007.

[26] MARTENA M., BOTTO, D., FINO, P., SABBADINI, S., GOLA, M.M., BADINI, C. Modelling of TBC system failure: Stress distribution as a function of TGO thickness and thermal expansion mismatch. Engineering Failure Analysis, v. 13, p. 409-426, 2006.

[27] EVANS, A. G., CLARKE, D.R., LEVI, C. G. The influence of oxides on the performance of advanced gas turbines. Journal of the European Ceramic Society, v. 28, p.1405-1419, 2008.

[28] JOHNSON, C. A., RUUD, J. A., BRUCE, R. AND WORTMAN, D. Relationships between residual stress, microstructure and mechanical properties of electron beam physical vapor deposition thermal barrier coatings. Surface and Coatings Technoogy, v. 108-109, p.80-85, 1998.

[29] GREGORI, G., LI, L., NYCHKA, J. A. AND CLARKE, D. R. Vibration damping of superalloys and thermal barrier coatings at high-temperatures. Materials Science and Engineering A, v. 466, p. 256-264, 2007.

[30] STRANGMAN, T. E. Thermal barrier coatings for turbine airfoils. Thin Solid Films, v. 127, p.93-105, 1985.

[31] TAYLOR, T. A., US Patent 5,073,433, 1991.

[32] BRUCE, R. W. AND SCHAEFFER, J. C., European Patent EP1281788, 2003.

[33] WU, B. C. et al. Degradation mechanisms of $\mathrm{ZrO}_{2}-8 \%$ $\mathrm{Y}_{2} \mathrm{O}_{3} / \mathrm{Ni}-22 \mathrm{Cr}-10 \mathrm{Al}-1 \mathrm{Y}$ thermal barrier coatings. Journal of the American Ceramic Society, v.72, n.2, p.212-218, 1989.

[34] SOBOLEV, V.V., GUILEMANY, J.M., NUTTING, J. High velocity oxy-fuel spraying: theory, structure-property relationships and applications. Maney Publishing, London, UK, 2004, 397 p.

[35] LI, Y, LI, C.J., ZHANG, Q., YANG, G.J., LI, C.X. Influence of TGO Composition on the Thermal Shock Lifetime of Thermal Barrier Coatings with Cold-sprayed MCrAlY Bond Coat. Journal of Thermal Spray Technology, v. 19, n. 1-2, p.168-177, 2009.

[36] ZHANG, Q., LI, C.J., LI, Y., ZHANG, S.L., WANG, X.R., GUAN-JUN YANG, G.J., LI, C.X. Thermal Failure of Nanostructured Thermal Barrier Coatings with Cold-Sprayed Nanostructured NiCrAlY Bond Coat. Journal of Thermal Spray Technology, V. 17, N. 5-6, p. 838-845, 2008.

[37] CHEN, W.R., WU, X., MARPLE, B.R., NAGY, D.R., PATNAIK, P.C. TGO growth behaviour in TBCs with APS and HVOF bond coats. Surface and Coatings Technology, v. 202, n.12, p. 2677-2683, 2008.

[38] NI, L. Y., LIU, C., HUANG, H., ZHOU, C. G. Ther- mal Cycling Behavior of Thermal Barrier Coatings with HVOF NiCrAlY Bond Coat. Journal of Thermal Spray Technology, v. 20, n. 5, p. 1133-1138, 2011.

[39] ZHANG, Y.; WANG, Y.; YIN, K.; XU, H. Finite element analysis of residual stresses in Zirconia thermal barrier coatings on superalloy. Journal of the Ceramic Society of Japan, v.S112, n.5, p.1122-1224, 2004.

[40] DORFMAN, M.R.; KUSHNER, B.A., ROTOLICO, A.J. A review of thermal barrier coatings for diesel engine applications. Metco /Perkin Elmer Int. Com., New York, 1991, 19p.

[41] DEMASI-MARCIN, J.T., GUPTA, D.K. Protective coatings in the gas turbine engine. Surface and Coatings Technology, v.68/69, p.1-9, 1994.

[42] COSACK, T.; PAWLOWSKI, L.; SCHINEIDERBANGER, S.; STURLESE, S. Thermal barrier coatings on turbine blades by plasma spraying with improved cooling. Transactions of the ASME - Journal of Engineering for Gas Turbine and Power, v. 1, n. 6, p.272-276, 1994.

[43] XIE, L., SOHN, Y., JORDAN, E.H., GELL, M. The effect of bond coat grit blasting on the durability and thermally grown oxide stress in an electron beam physical vapor deposited thermal barrier coating. Surface and Coatings Technology, v.176, n.1, p. 57-66, 2003.

[44] BACH, W., ENGL, L., BACH, C., LUGSCHEIDER, E., PARCO, M., DUDA, T. Evaluation of modern HVOF systems concerning the application of hot corrosion protective coatings. Thermal Spray 2003: advancing the science and applying the technology, C. Moreau and B. Marple Ed., ASM international, Materials Park, OH, 2003, p. 519-527

[45] LIMA, C.; NIN, J.; GUILEMANY, J. Evaluation of residual stresses of thermal barrier coatings with HVOF thermally sprayed bond coats using the Modified Layer Removal Method (MLRM). Surface and Coatings Technology, Holanda, v. 200, p. 5963-5972, 2006.

[46] NEUER, G. et al. Some aspects of properties design of plasma-sprayed thermal barrier coatings. Powder Metallurgy International, v.23, n.2, p. 108-111, 1991.

\begin{tabular}{|l|l|l|l|}
\hline 9500153913 & $\mathrm{j}$ & ar & 2-s2.0-58149290 \\
\hline
\end{tabular}

[47] MOSKAL, G.; SWADZBA, L.; RZYCHON, T. Measurement of residual stress in plasma sprayed TBC whit a gradient of porosity and chemical composition. Journal of Achievements in Materials and Manufacturing Engineering, v.23, n.2, 2007, p.31-34.

[48] STEFFENS, H.D., BABLAK, Z., BRADL, W. Thermal barrier coatings: some aspects of properties design. Thermal Spray Coatings: Properties, Processes and Applications, T.F. Bernecki Ed. - ASM International, Ohio, 1991, p. 289-294.

[49] TSAI, H.L., TSAI, P.C. Performance of laser-glazed plasma-sprayed $\left(\mathrm{ZrO}_{2}-12 \mathrm{wt} . \% \mathrm{Y}_{2} \mathrm{O}_{3}\right) /(\mathrm{Ni}-22 \mathrm{wt} . \% \mathrm{Cr}-10 \mathrm{wt} . \% \mathrm{Al}-$ $1 \mathrm{~V} 4 . \% \mathrm{Y}$ ) thermal barrier coatings in cyclic oxidation tests. Surface and Coatings Technology, v.71, n. 1, p. 53-59, 1995.

[50] SWADŹBA, L., MOSKAL, G., MENDALA, B., HETMAŃCZYK, M. Character-ization of microstructure and properties of TBC systems with gradient of chemical composition and porosity. Archives of Metallurgy and Materials, v. 53, n. 3, p. $945-954,2008$. 
[51] COSACK, T.; PAWLOWSKI, L.; SCHINEIDERBANGER, S.; STURLESE, S. Thermal barrier coatings on turbine blades by plasma spraying with improved cooling. Transactions of the ASME - Journal of Engineering for Gas Turbine and Power, v. 1, n. 6, p.272-276, 1994.

[52] KUCUK, A., BERNDT, C.C., SENTURK, U., LIMA, R.S., LIMA, C.R.C. Influence of plasma spray parameters on mechanical properties of yttria stabilized zirconia coatings. I: Four point bend test. Materials Science and Engineering A, v. 284 , n. $1-2,2000$, p. 29-40.

[53] LIMA, C. R. C.; GUILEMANY, J. M. Adhesion improvements of Thermal Barrier Coatings with HVOF thermally sprayed bond coats. Surface and Coatings Technology, v. 201, n.8, p.4694-4701, 2007.

[54] YAMAZAKI, Y.; FUKANUMA, H.; OHNO, N. Effect of interfacial roughness of bond coat on the residual adhesion strength of a plasma sprayed TBC system after thermal cycle fatigue. Journal of Solid Mechanics and Materials Engineering, v. 4, n.2, p. 196-207, 2010.

[55] KNIGHT, R., ZHANGXIONG, D., KIM, E.H., SMITH, R.W., SAHOO, P., BUCCI, D. Influence of bond coat surface characteristics on the performance of tbc systems. Thermal Spray: Meeting the Challenges of the $21^{\text {st }}$ Century, Christian Coddet Ed., ASM International, Ohio, USA, 1998, p.1549-1554. [56] NIKI, T.; OGAWA, K.; SHOJI, T. Mechanical and high temperature oxidation properties of cold sprayed CoNiCrAlY coatings for Thermal Barrier Coating. Journal of Solid Mechanics and Materials Engineering, v.2, n.6, p. 739-747, 2008.

[57] LIMA, C.R.C., CINCA, N., GUILEMANY, J.M. Study of the high temperature oxidation performance of Thermal Barrier Coatings with HVOF sprayed bond coat and incorporating a PVD ceramic interlayer. Ceramics International, v. 38, n. 8, p. 6423-6429, 2012.

[58] KARLSSON, A. M.; HUTCHINSON, J.W.; EVANS, A.G. The displacement of the thermally grown oxide in thermal barrier systems upon temperature cycling. Materials Science and Engineering, A, v. 351, p.244-257, 2003.

[59] EVANS, A. G., CLARKE, D.R., LEVI, C. G. The influence of oxides on the performance of advanced gas turbines. Journal of the European Ceramic Society, v. 28, p.1405-1419, 2008.

[60] MARTENA M., BOTTO, D., FINO, P., SABBADINI, S., GOLA, M.M., BADINI, C. Modelling of TBC system failure: Stress distribution as a function of TGO thickness and thermal expansion mismatch. Engineering Failure Analysis, v. 13, p. 409-426, 2006.

[61] SWADŹBA, R., WIEDERMANN, J., HETMAŃCZYK, M., SWADŹBA, L., WITALA, B., MOSKAL, G., MENDALA, B., KOMENDERA, L. Microstructural examination of TGO formed during pre-oxidation on Pt-aluminized Ni-based superalloy. Materials and Corrosion, v. 65, n. 3, p. 319-323, 2014.

[62] KITAZAWA, R., KAKISAWA, H., KAGAWA, Y. Anisotropic TGO morphology and stress distribution in EBPVD $\mathrm{Y}_{2} \mathrm{O}_{3}-\mathrm{ZrO}_{2}$ thermal barrier coating after in-phase thermomechanical test. Surface and Coatings Technology, v. 238, n. 15, p. 68-74, 2014.

[63] FRITSCHER, K. , BRAUE, W., SCHULZ, U. Assessment of cyclic lifetime of NiCoCrAlY/ZrO tems via reactive element enrichment in the mixed zone of the TGO scale. Metallurgical and Materials Transactions A: Physical Metallurgy and Materials Science, v. 44, n. 5, p. 2070-2082, 2013.

[64] OKADA, M., VASSEN, R., KARGER, M., SEBOLD, D., MACK, D., JARLIGO, M.O., BOZZA, F. Deposition and oxidation of oxide-dispersed CoNiCrAlY bondcoats. Journal of Thermal Spray Technology,

\begin{tabular}{|l|l|l|l|}
\hline 24537 & $\mathrm{j}$ & ar & 2-s2.0-84890856 \\
\hline
\end{tabular}

v. 23, n. 1-2, p. 147-153, 2014.

[65] PAN, W., PHILLPOT, S.R., WAN, C., CHERNATYNSKIY, A., QU, Z. Low thermal conductivity oxides. MRS Bulletin, v. 37, p. 917-922, 2012.

[66] BOBZIN, K., BAGCIVAN, N., BRÖGELMANN, T., YILDIRIM, B. Influence of temperature on phase stability and thermal conductivity of single- and double-ceramic-layer EB-PVD TBC top coats consisting of $7 \mathrm{YSZ}, \mathrm{Gd}_{2} \mathrm{Zr}_{2} \mathrm{O}_{7}$ and $\mathrm{La}_{2} \mathrm{Zr}_{2} \mathrm{O}_{7}$. Surface and Coatings Technology, v. 237, n. 25, p. $56-64,2013$.

[67] XU, Z., ZHONG, X., ZHANG, J., ZHANG, Y., CAO, X., HE, L. Effects of deposition conditions on composition and thermal cycling life of lanthanum zirconate coatings. Surface and Coatings Technology, v. 202, n. 19, p. 4714-4720, 2008. [68] MA, W., DONG, H., GUO, H., GONG, S., ZHENG, X. Thermal cycling behavior of $\mathrm{La}_{2} \mathrm{Ce}_{2} \mathrm{O}_{7} / 8 \mathrm{YSZ}$ double-ceramiclayer thermal barrier coatings prepared by atmospheric plasma spraying. Surface and Coatings Technology, v. 204, n. 21-22, p. 3366-3370, 2010.

[69] MATSUMOTO, M. Development of plasma sprayed thermal barrier coatings with low thermal conductivity and high oxidation resistance. Journal of the Ceramic Society of Japan, v.115, n.2, 2007, p.118-123.

[70] RACEK, O.; BERNDT, C.C. Mechanical property variations within thermal barrier coatings. Surface and Coatings Technology, v.202, n.2, p.362-369, 2007.

[71] MAUREL, V.; BODMAN, P.; RÉMY, L. Influence of substrate strain anisotropy in TBC system failure. Surface and Coatings Technology, v.206, n.7, p. 1634-1639, 2011.

[72] LEVI, C.G., HUTCHINSON, J.W., VIDAL-SÉTIF, M.H., JOHNSON, C.A. Environmental degradation of thermalbarrier coatings by molten deposits. MRS Bulletin, v. 37, n. 10, p. 932-941, 2012.

[73] PUJOL, G., ANSART, F., BONINO, J.-P., MALIÉ, A., HAMADI, S. Step-by-step investigation of degradation mechanisms induced by CMAS attack on YSZ materials for TBC applications. Surface and Coatings Technology, v. 237, n. 25, p. 71-78, 2013.

[74] MOHAN, P., PATTERSON, T., YAO, B., SOHN, Y. Degradation of thermal barrier coatings by fuel impurities and CMAS: Thermochemical interactions and mitigation approaches. Journal of Thermal Spray Technology, v. 19, n. 1-2, p. 156-167, 2010.

[75] DREXLER , J.M. GLEDHILL , A.D. SHINODA , K.. VASILIEV , A.L REDDY , K.M. SAMPATH , S., PADTURE, N.P. Jet Engine Coatings for Resisting Volcanic Ash Damage, 
Advanced Materials, v. 23, n. 21, p. 2419-2424, 2011.

\begin{tabular}{|l|l|l|l}
\hline$\cdot \cdot$ & $\mathrm{i}$ & ar & 2-s2.0-84889095 \\
\hline
\end{tabular}

[76] FEUERSTEIN, A., KNAPP, J., TAYLOR, T., ASHARY, A., BOLCAVAGE, A., HITCHMAN. N. Technical and Economical Aspects of Current Thermal Barrier Coating Systems for Gas Turbine Engines by Thermal Spray and EBPVD: A Review. Journal of Thermal Spray Technology, v. 17, n. 2, p. 199-213, 2008.

[77] HARDWICKE, C.U., LAU, Y.C. Advances in Thermal Spray Coatings for Gas Turbines and Energy Generation: A Review. Journal of Thermal Spray Technology, v. 22, n. 5, p. 564576, 2013.

[78] KROGSTAD, J.A., LEPPLE, M., LEVI, C.G. Opportunities for improved TBC durability in the $\mathrm{CeO}_{2}-\mathrm{TiO}_{2}-\mathrm{ZrO}_{2}$ system. Surface and Coatings Technology, v. 221, p. 44-52, 2013. [79] SAMPATH, S., SCHULZ, U., JARLIGO, M.O., KURODA, $\mathrm{S}$. Processing science of advanced thermal-barrier systems. MRS Bulletin, v. 37, p. 903-910, 2012.

[80] VAßEN, R., KAGAWA, Y., SUBRAMANIAN, R., ZOMBO, P., ZHU, D. Testing and evaluation of thermal-barrier coatings. MRS Bulletin, v. 37, p. 911-916, 2012. 